\title{
Inverses and Determinants of Toeplitz-Hessenberg Matrices
}

\author{
Roksana Słowik
}

\begin{abstract}
The inverses of Toeplitz-Hessenberg matrices are investigated. It is known that each inverse of such a matrix is a sum of a lower triangular matrix $L$ and a matrix $R$ of rank 1. The formulas of $L$ and $x, y$ such that $x y^{T}=R$ are derived. Using this result we propose an algorithm for inverting Toeplitz-Hessenberg matrices. Moreover, from the expression of the inverse a formula for the determinant is deduced.
\end{abstract}

\section{Introduction}

The Hessenberg (or lower Hessenberg) matrices are the matrices $H=\left[h_{i j}\right]$ satisfying condition $h_{i j}=0$ for $j-i>1$. More general, the matrix is said to be $k$-Hessenberg if and only if $H_{i j}=0$ for $j-i>k$.

Asplund $[3$ was probably the first who discovered that a nonsingular matrix is strictly 1-Hessenberg if and only if its inverse is a sum of a matrix of rank one and a lower triangular matrix with zeros in the main diagonal. Analogous theorem is true for $k$ Hessenberg matrices. It is interesting that even more can be said: the right upper block of the inverse of $k$-Hessenberg matrix can be written as a product of $n \times k$ and $k \times n$ matrices [10]. Moreover, it turned out that also inverses of $\mathbb{N} \times \mathbb{N}$ Hessenberg matrices (on the condition they exist) can also be written as such sums 22. This property of inverses was discussed most intensively for the tridiagonal matrices [4 6]. Clearly, such representation of inverses can result in proposing algorithms for finding its entries 12.

In this paper we would like to continue the discussion on this subject and assuming that $H^{-1}=L-\frac{1}{\gamma} x y^{T}$ (where $L$ is lower triangular and $x, y$ are vectors) present the formulas for $L, x$ and $y$. Clearly, when the entries of $H$ are arbitrarily chosen it is very complicated. Therefore, we limit ourselves to the class of Toeplitz-Hessenberg matrices,

Received August 16, 2017; Accepted January 31, 2018.

Communicated by Ren-Cang Li.

2010 Mathematics Subject Classification. 15A09, 15A15, 15B05, 65F05.

Key words and phrases. Hessenberg matrix, Toeplitz matrix, matrix inverse, determinant. 
i.e., matrices of the form

$$
H=H_{n}=\left[\begin{array}{cccccc}
a_{0} & a_{-1} & & & & \\
a_{1} & a_{0} & a_{-1} & & & \\
a_{2} & a_{1} & a_{0} & a_{-1} & & \\
\vdots & & & \ddots & & \\
a_{n-2} & a_{n-3} & \ldots \ldots \ldots & a_{0} & a_{-1} \\
a_{n-1} & a_{n-2} & \ldots \ldots \ldots & a_{1} & a_{0}
\end{array}\right]_{n \times n}
$$

We will assume that $H$ is strictly Hessenberg, i.e., $a_{-1} \neq 0$. The first result we are going to prove in this paper is the following.

Theorem 1.1. Let $H$ be an invertible matrix of form $(1.1)$, where $a_{-1} \neq 0$. Then $H$ is invertible if and only if

$$
\gamma=a_{n-2}-a_{n-1}-\sum_{i=2}^{n-1} a_{n-i-1}\left(\sum_{j=1}^{i-1} a_{j-1} b_{i-j-1}\right) \neq 0
$$

and in this case

$$
H^{-1}=\left[\begin{array}{cc}
0 & 0 \\
\widetilde{A} & 0
\end{array}\right]-\frac{1}{\gamma} x_{H} y_{H}^{T}
$$

where

$$
\begin{aligned}
& \widetilde{A}_{i j}=\left\{\begin{array}{ll}
b_{i-j} & \text { if } i \geq j, \\
0 & \text { otherwise, }
\end{array} \quad \text { with } b_{0}=1, b_{k}=-\frac{1}{a_{-1}} \sum_{r=0}^{k-1} a_{r} b_{k-r-1} \text { for } k \geq 1,\right. \\
& \left(x_{H}\right)_{i}= \begin{cases}1 & \text { if } i=1 \\
-\sum_{j=1}^{i-1} a_{j-1} b_{i-j-1} & \text { if } 1<i \leq n\end{cases} \\
& \left(y_{H}\right)_{i}=\left(x_{H}\right)_{n+1-i}= \begin{cases}-\sum_{j=i}^{n-1} a_{n-j-1} b_{j-1} & \text { if } 1 \leq i<n, \\
1 & \text { if } i=n .\end{cases}
\end{aligned}
$$

Based on this theorem we propose an algorithm for inverting Toeplitz-Hessenberg matrices. Moreover, we will present one more conclusion following from Theorem 1.1. It is natural that in some formulas and algorithms for finding inverses one may sometimes use submatrices and subminors. For instance in [1,7] there are formulas for the entries of $H^{-1}$ that involve the minors of $H$. In Section 3 we will present some other formula for the inverse of a Toeplitz-Hessenberg matrix and comparing it with Theorem 1.1 we will obtain some formula for $\operatorname{det}\left(H_{n}\right)$. 


\section{Inverses}

Before we start, let's introduce some notation. By $H_{n}$ or $H$ we will mean a matrix of form (1.1). The symbol $\mathcal{M}_{n \times m}(F)$ will stand for the set of $n \times m$ matrices over a (commutative) field $F$, and we will abbreviate $\mathcal{M}_{n \times n}(F)$ to $\mathcal{M}_{n}(F)$. $I_{n}$ will denote the $n \times n$ identity matrix, whereas $0_{n \times m}$ the $n \times m$ zero matrix. Moreover, let $E_{k m}$ be a matrix with 1 in the position $(k, m)$ and 0 in every other position (in the case of using this symbol the dimension of $E_{k m}$ will follow from the context). We will also abbreviate $\left(x_{H}\right)_{i}\left(\left(y_{H}\right)_{i}\right.$ respectively) to $x_{H, i}$.

To prove Theorem 1.1 we are going to use the result from 11 where more precise expressions for $L, x$ and $y$ are presented. Namely, we need the following theorem.

Theorem 2.1. [11, Theorem 3.1] Let $H$ be a strict $k$-Hessenberg matrix with the block decomposition

$$
H=\left[\begin{array}{ll}
B & A \\
D & C
\end{array}\right], \quad A \in \mathcal{M}_{n-k}(F), B \in \mathcal{M}_{(n-k) \times k}(F), C \in \mathcal{M}_{k \times(n-k)}(F), D \in \mathcal{M}_{k}(F) .
$$

Then $H$ is invertible if and only if $C A^{-1} B-D$ is invertible and if $H$ is invertible we have

$$
H^{-1}=\left[\begin{array}{cc}
0 & 0 \\
A^{-1} & 0
\end{array}\right]-\left[\begin{array}{c}
I_{k} \\
-A^{-1} B
\end{array}\right]\left(C A^{-1} B-D\right)^{-1}\left[\begin{array}{ll}
-C A^{-1} & I_{k}
\end{array}\right] .
$$

For $k=1$ the blocks $A, B, C, D$ are

$$
A=A_{n}=\left[\begin{array}{cccccc}
a_{-1} & & & & \\
a_{0} & a_{-1} & & & \\
a_{1} & a_{0} & a_{-1} & & \\
\vdots & & \ddots & \ddots & \\
a_{n-4} & a_{n-5} & \cdots & a_{0} & a_{-1} \\
a_{n-3} & a_{n-4} & \cdots & a_{1} & a_{0} & a_{-1}
\end{array}\right]_{(n-1) \times(n-1)},
$$

Note that $\left(B^{T}\right)_{i}=C_{n-i-1}$. This fact is going to be useful later on. Formula (2.1) can be simplified to

$$
\begin{aligned}
H^{-1} & =\left[\begin{array}{cr}
0 & 0 \\
A^{-1} & 0
\end{array}\right]-\frac{1}{C A^{-1} B-D}\left[\begin{array}{c}
1 \\
-A^{-1} B
\end{array}\right]\left[\begin{array}{ll}
-C A^{-1} & 1
\end{array}\right] \\
& =\left[\begin{array}{cr}
0 & 0 \\
A^{-1} & 0
\end{array}\right]-\frac{1}{C A^{-1} B-D}\left[\begin{array}{cc}
-C A^{-1} & 1 \\
-A^{-1} B C A^{-1} & -A^{-1} B
\end{array}\right] .
\end{aligned}
$$


First let's focus on determining $A^{-1}$. For any $1 \leq k \leq n-1$, let $C_{n-1}^{(k)}(A)$ denote the matrix $I_{n-1}+\sum_{i=k+1}^{n-1} \frac{a_{i-k-1}}{a_{-1}} E_{i k}$. Additionally, let $C_{n-1}^{(0)}(A)$ denote $I_{n-1}$. We can observe the following.

Remark 2.2. Let $A$ be given as in 2.2 . Then $A=a_{-1} \prod_{k=1}^{n-2} C_{n-1}^{(k)}(A)$.

The decomposition from Remark 2.2 can be used to find the inverse of $A$. First we find the inverses of $C_{n-1}^{(k)}(A)$. Namely, one can check that they are given in

Remark 2.3. Let $n, k \in \mathbb{N}, n \geq 3, \alpha \in F \backslash\{0\}$, and let $A$ be given as in 2.2). Then

$$
\left[C_{n-1}^{(k)}(A)\right]^{-1}=I_{n-1}-\sum_{r=k+1}^{n-1} a_{i-k-1} E_{i k} .
$$

From Remarks 2.2 and 2.3 we get now the form of $A^{-1}$.

Lemma 2.4. Let $A$ be given as in (2.2) with $n>3$. Then

$$
A^{-1}=\frac{1}{a_{-1}} \sum_{k=0}^{n-2} \sum_{r=1}^{n-1-k} b_{k} E_{r+k, r}
$$

where $b_{0}=1$ and $b_{k}=-\frac{1}{a_{-1}} \sum_{r=0}^{k-1} a_{r} b_{k-r-1}$ for $k \geq 1$.

Proof. Since $A$ is a triangular Toeplitz matrix, so is $A^{-1}$. We prove 2.3 inductively on $n$. We have

$$
\left[\begin{array}{ccc}
1 & & \\
0 & 1 & \\
0 & -\frac{a_{0}}{a_{-1}} & 1
\end{array}\right]\left[\begin{array}{ccc}
1 & & \\
-\frac{a_{0}}{a_{-1}} & 1 & \\
-\frac{a_{1}}{a_{-1}} & 0 & 1
\end{array}\right]=\left[\begin{array}{ccc}
1 & & \\
-b_{1} & 1 & \\
b_{2} & b_{1} & 1
\end{array}\right]
$$

with

$$
b_{1}=-\frac{a_{0}}{a_{-1}}, \quad b_{2}=\left(\frac{a_{0}}{a_{-1}}\right)^{2}-\frac{a_{1}}{a_{-1}}=-\frac{1}{a_{-1}}\left(a_{0} b_{1}+a_{1} b_{0}\right) .
$$

The first step of induction holds.

Consider now $n>4$. We have

$$
\begin{aligned}
& {\left[\begin{array}{c|cc|ccc}
1 & & & & \\
\hline 0 & 1 & & & \\
0 & b_{1} & 1 & & \\
\vdots & \vdots & & \ddots & \\
0 & b_{n-3} & \cdots & b_{1} & 1
\end{array}\right]\left[\begin{array}{ccc|ccc}
1 & & & \\
\hline-\frac{a_{0}}{a_{-1}} & 1 & & & \\
-\frac{a_{1}}{a_{-1}} & & 1 & & \\
\vdots & & \ddots & \\
-\frac{a_{n-3}}{a_{-1}} & & & 1
\end{array}\right]=\left[\begin{array}{ccccc}
1 & & & \\
\hline b_{1} & 1 & & \\
b_{2} & b_{1} & 1 & \\
\vdots & \vdots & & \ddots & \\
& & & & \\
b_{n-2} & b_{n-3} & \cdots & b_{1} & 1
\end{array}\right],} \\
& b_{n-2}=-\frac{a_{0}}{a_{-1}} b_{n-3}-\frac{a_{1}}{a_{-1}} b_{n-4}-\cdots-\frac{a_{n-4}}{a_{-1}} b_{1}-\frac{a_{n-3}}{a_{-1}} b_{0} \text {. }
\end{aligned}
$$


As it was mentioned before, from Theorem 1.1 it follows from the fact that $H^{-1}$ can be represented as $L-\frac{1}{\gamma} x y^{T}$. Clearly, by Theorem 2.1 in our case $x, y$ are of the forms:

$$
x_{H}=\left[\begin{array}{c}
1 \\
-A^{-1} B
\end{array}\right], \quad y_{H}=\left[\begin{array}{ll}
-C A^{-1} & 1
\end{array}\right] .
$$

Thus, we can give the following proof.

Proof of Theorem 1.1. Let $2 \leq i \leq n$. Then

$$
x_{H, i}=\left(-A^{-1} B\right)_{i-1}=-\sum_{j=1}^{i-1}\left(A^{-1}\right)_{i-1, j} \cdot B_{j}=-\sum_{j=1}^{i-1} b_{i-1-j} a_{j-1} .
$$

To find $\left(C A^{-1}\right)_{i}$ for $1 \leq i \leq n-1$ we can either perform the same calculations for $A^{-1} B$ or make use of the following two facts:

(1) $B_{i}^{T}=C_{n+1-i}$

(2) the $i$-th entry of the $k$-th column of $A^{-1}$ is equal to the $(n-1-i)$-th entry of $(n-1-i)$-th row of $A^{-1}$.

From the first of these two ways we get the formula for $y_{i}$ that appears in Theorem 1.1 . From the second one we obtain the equality $y_{i}=x_{n+1-i}$.

We finish the proof by determining $C A^{-1} B-D$ :

$$
\begin{aligned}
C A^{-1} B-D & =\sum_{i=2}^{n} C_{i}\left(A^{-1} B\right)_{i}-D \\
& =a_{n-2}-\sum_{i=1}^{n-1}\left[a_{n-i-1} \cdot\left(\sum_{j=1}^{i-1} a_{j-1} b_{i-j-1}\right)\right]-a_{n-1} .
\end{aligned}
$$

From Theorem 1.1 we derive the following algorithm for calculating $H^{-1}$.
Algorithm 2.5. (1) let $H=[0]_{n \times n}$;
$b_{k}=b_{k}+a_{r} b_{k-r-1}$,
(2) for $k=2,3, \ldots, n$ put $H_{k k}^{\prime}=\frac{1}{a_{-1}}$;
$b_{k}=-\frac{1}{a_{-1}} b_{k}$,
for $r=0,1, \ldots, k-1$
(3) put $b_{0}=1$,
$H_{k+r+2, k+1}^{\prime}=b_{r+1}$,
for $k=1,2, \ldots, n-2$
(4) $x_{1}=1$,
$b_{k}=0$,
for $i=2,3, \ldots, n-1$
for $r=0,1, \ldots, k-1$
$x_{i}=0$, 


$$
\begin{aligned}
& \text { for } j=1,2, \ldots, i-1 \\
& x_{i}=x_{i}-a_{j-1} b_{i-j-1},
\end{aligned}
$$

(5) $\gamma=0$,

for $i=2,3, \ldots, n-1$

$\gamma=\gamma+a_{n-i-1} x_{i}$,

$$
\gamma=a_{n-2}-a_{n-1}-\gamma,
$$

(6) for $i=1,2, \ldots, n$

$$
\begin{aligned}
& \text { for } j=1,2, \ldots, n \\
& H_{i j}^{\prime}=H_{i j}^{\prime}-\frac{1}{\gamma} x_{i} x_{n+1-j} .
\end{aligned}
$$

\section{Determinants of Toeplitz-Hessenberg matrices}

It is known that the determinant of $H_{n}$ can be evaluated using the Trudi formula (see [8,9]). Namely,

$$
\operatorname{det} H_{n}=\sum_{k_{1}+2 k_{2}+\cdots+n k_{n}=n}\left(\begin{array}{c}
k_{1}+k_{2}+\cdots+k_{n} \\
k_{1}, k_{2}, \ldots, k_{n}
\end{array}\right)\left(-a_{-1}\right)^{n-k_{1}-\cdots-k_{n}} a_{0}^{k_{1}} a_{1}^{k_{2}} \cdots a_{n-1}^{k_{n}} .
$$

In the present section we will obtain some other formula for $\operatorname{det}\left(H_{n}\right)$. As it contains some multiple sums, in comparison to (3.1) it may seem more complicated. However, its advantage is that it does not involve the partitions of $n$. Let's copy from [1] a formula for the entries of $\left(H^{-1}\right)_{i j}$ that the authors have concluded from the Cayley formula and the Sylvester theorem on determinants:

$$
\left(H^{-1}\right)_{i j}=(-1)^{i+j}\left(\prod_{k=0}^{i-j-1} h_{i-k, i-k+1}\right) \cdot \frac{\operatorname{det} H_{1,2, \ldots, j-1}^{1,2, \ldots, j-1} \cdot \operatorname{det} H_{i+1, i+2, \ldots, n}^{i+1, i+2, \ldots, n}}{\operatorname{det} H}
$$

where $H_{l_{1}, \ldots, l_{m}}^{k_{1}, \ldots, k_{m}}$ denotes the submatrix of $H$ consisting of the entries standing in the intersections of the rows $k_{1}, \ldots, k_{m}$ and columns $l_{1}, \ldots, l_{m}$.

Using (3.2) we will prove the following theorem.

Theorem 3.1. Let $H_{n}$ be defined as in (1.1) with $a_{-1} \neq 0$. Then

$$
\operatorname{det}\left(H_{n}\right)=\left(-a_{-1}\right)^{n-1} \cdot\left\{b_{n-1}-\frac{\left(\sum_{j=1}^{n-2} a_{j-1} b_{n-j-2}\right)^{2}}{a_{n-2}-a_{n-1}-\sum_{i=2}^{n-1} a_{n-i-1}\left(\sum_{j=1}^{i-1} a_{j-1} b_{i-j-1}\right)}\right\}^{-1} .
$$

Proof. Since we are dealing with Toeplitz-Hessenberg matrices, the submatrices of $H_{n}$ consisting of some $k$ consecutive rows and columns are simply the matrices $H_{k}$. Moreover, since all the entries $h_{i-k, i-k+1}$ lie in the first superdiagonal, in this case we have $h_{i-k, i-k+1}=a_{-1}$ for all $i, k$. Thus 3.2 can be transformed into

$$
\left(H_{n}^{-1}\right)_{i j}=\frac{(-1)^{i+j} a_{-1}^{i-j} \operatorname{det}\left(H_{j-1}\right) \operatorname{det}\left(H_{n-i}\right)}{\operatorname{det}\left(H_{n}\right)} .
$$


Substituting $i=n, j=1$ we obtain the equality: $\operatorname{det}\left(H_{n}\right)=\frac{\left(-a_{-1}\right)^{n-1}}{\left(H_{n}^{-1}\right)_{n 1}}$. Now it is a natural idea to use Theorem 1.1. This leads to

$$
\begin{aligned}
\operatorname{det}\left(H_{n}\right) & =\frac{\left(-a_{-1}\right)^{n-1}}{\left(H_{n}^{-1}\right)_{n 1}}=\frac{\left(-a_{-1}\right)^{n-1}}{b_{n-2}-\frac{1}{\gamma} x_{n} y_{1}}=\frac{\left(-a_{-1}\right)^{n-1}}{b_{n-2}-\frac{x_{n}^{2}}{\gamma}} \\
& =\left(-a_{-1}\right)^{n-1} \cdot\left\{b_{n-2}-\frac{\left(\sum_{j=1}^{n-2} a_{j-1} b_{n-j-2}\right)^{2}}{a_{n-2}-a_{n-1}-\sum_{i=2}^{n-1} a_{n-i-1}\left(\sum_{j=1}^{i-1} a_{j-1} b_{i-j-1}\right)}\right\}^{-1}
\end{aligned}
$$

Obviously, 3.3 used for various $i, j$ can lead to some combinatorial identities. One example is presented below.

Corollary 3.2. If $a_{0}, a_{1}, \ldots, a_{n-1}$ are arbitrary numbers, then

$$
\begin{gathered}
\sum_{k_{1}+2 k_{2}+\cdots+n k_{n}=n}\left(\begin{array}{c}
k_{1}+\cdots+k_{n} \\
k_{1}, \ldots, k_{n}
\end{array}\right)(-1)^{n-k_{1}-\cdots-k_{n}} a_{0}^{k_{1}} a_{1}^{k_{2}} \cdots a_{n-1}^{k_{n}} \\
=\left(-a_{-1}\right)^{n-1} \cdot\left\{b_{n-2}-\frac{\left(\sum_{j=1}^{n-2} a_{j-1} b_{n-j-2}\right)^{2}}{a_{n-2}-a_{n-1}-\sum_{i=2}^{n-1} a_{n-i-1}\left(\sum_{j=1}^{i-1} a_{j-1} b_{i-j-1}\right)}\right\}^{-1} .
\end{gathered}
$$

\section{References}

[1] J. Abderramán Marrero and V. Tomeo, On the closed representation for the inverses of Hessenberg matrices, J. Comput. Appl. Math. 236 (2012), no. 12, 2962-2970.

[2] J. Abderramán Marrero, V. Tomeo and E. Torrano, On inverses of infinite Hessenberg matrices, J. Comput. Appl. Math. 275 (2015), 356-365.

[3] E. Asplund, Inverses of matrices $\left\{a_{i j}\right\}$ which satisfy $a_{i j}=0$ for $j>i+p$, Math. Scand. 7 (1959), 57-60.

[4] B. Bukhberger and G. A. Emel'yanenko, Methods of inverting tridiagonal matrices, USSR Comput. Math. and Math. Phys. 13 (1973), no. 3, 10-20.

[5] D. K. Faddeev, Some properties of a matrix that is the inverse of a Hessenberg matrix, Numerical Methods and Questions in the Organization of Calculations 5, Zap. Nauchn. Sem. Leningrad. Otdel. Mat. Inst. Steklov. (LOMI) 111 (1981), 177-179.

[6] Y. Ikebe, On inverses of Hessenberg matrices, Linear Algebra Appl. 24 (1979), 93-97.

[7] J. Maroulas, Factorization of Hessenberg matrices, Linear Algebra Appl. 506 (2016), 226-243. 
[8] M. Merca, A note on the determinant of a Toeplitz-Hessenberg matrix, Spec. Matrices 2 (2014), 10-16.

[9] T. Muir, A Treatise on the Theory of Determinants, Revised and enlarged by William H. Metzler, Dover, New York, 1960.

[10] M. J. Piff, Inverses of banded and $k$-Hessenberg matrices, Linear Algebra Appl. 85 (1987), 9-15.

[11] L. Verde-Star, Elementary triangular matrices and inverses of $k$-Hessenberg and triangular matrices, Spec. Matrices 3 (2015), 250-256.

[12] X. Zhong, On inverses and generalized inverses of Hessenberg matrices, Linear Algebra Appl. 101 (1988), 167-180.

Roksana Słowik

Silesian University of Technology, Institute of Mathematics, Kaszubska 23, 44-100

Gliwice, Poland

E-mail address: roksana.slowik@gmail.com 